\title{
Absorption of toluene using deep eutectic solvents: Quantum chemical calculation and experimental investigation
}

\author{
Yunfei Song, Shuo Chen, Fei Luo, Lanyi Sun*
}

State Key Laboratory of Heavy Oil Processing, College of Chemical

Engineering, China University of Petroleum (East China), Qingdao Shandong

266580, China 
Table S1. Abbreviations (Abb.) and chemical structures of HBAs studied

\begin{tabular}{ll}
\hline Abb. & Description \\
\hline A1 & Tetraethylammonium Chloride \\
A2 & Trimethylamine hydrochloride \\
A5 & Tetramethylammonium chloride \\
A6 & Methyl(triphenyl)phosphonium chloride \\
A8 & Choline chloride
\end{tabular}


Table S2. Abbreviations (Abb.) and chemical structures of HBDs studied

\begin{tabular}{|c|c|c|}
\hline Abbr. & Description & Chemical structures \\
\hline D1 & Benzoic acid & \\
\hline D2 & Capric acid & \\
\hline D3 & Hexanoic acid & \\
\hline D4 & Linoleic acid & \\
\hline D5 & Phenylacetic acid & \\
\hline D6 & Oleic acid & \\
\hline D7 & Sulfolane & \\
\hline D8 & Stearic acid & \\
\hline
\end{tabular}


Table S3. Infinite diluted solubility of toluene $\left(\mathrm{Sol}^{\infty}\right)$ in DESs predicted by COSMO-

SAC

\begin{tabular}{|c|c|c|c|c|}
\hline HBA & HBD & $\operatorname{Sol}^{\infty}(1: 1)$ & $\operatorname{Sol}^{\infty}(1: 2)$ & $\operatorname{Sol}^{\infty}(1: 3)$ \\
\hline $\mathrm{A} 1$ & D1 & 0.47376 & 0.43127 & 0.42793 \\
\hline A1 & D2 & 0.70038 & 0.73012 & 0.74067 \\
\hline A1 & D3 & 0.57826 & 0.56141 & 0.55951 \\
\hline $\mathrm{A} 1$ & D4 & 0.94358 & 1.03258 & 1.06053 \\
\hline A1 & D5 & 0.47762 & 0.42232 & 0.40601 \\
\hline A1 & D6 & 0.94627 & 1.02248 & 1.03845 \\
\hline $\mathrm{A} 1$ & D7 & 0.78814 & 0.7506 & 0.73486 \\
\hline A1 & D8 & 0.94111 & 1.00667 & 1.01528 \\
\hline A2 & D1 & 0.19361 & 0.2074 & 0.24057 \\
\hline A2 & D2 & 0.28668 & 0.39255 & 0.47305 \\
\hline A2 & D3 & 0.2202 & 0.2634 & 0.31026 \\
\hline $\mathrm{A} 2$ & D4 & 0.45006 & 0.6572 & 0.78642 \\
\hline A2 & D5 & 0.20721 & 0.2162 & 0.23828 \\
\hline A2 & D6 & 0.44465 & 0.64338 & 0.76249 \\
\hline A2 & D7 & 0.36679 & 0.3864 & 0.4158 \\
\hline A2 & D8 & 0.44895 & 0.64321 & 0.75569 \\
\hline A3 & D1 & 0.19361 & 0.14087 & 0.19748 \\
\hline A3 & D2 & 0.28668 & 0.34758 & 0.43559 \\
\hline A3 & D3 & 0.2202 & 0.20098 & 0.26869 \\
\hline A3 & D4 & 0.45006 & 0.62003 & 0.74311 \\
\hline $\mathrm{A} 3$ & D5 & 0.20721 & 0.13757 & 0.18516 \\
\hline A3 & D6 & 0.44465 & 0.60309 & 0.71535 \\
\hline A3 & D7 & 0.36679 & 0.20412 & 0.28034 \\
\hline A3 & D8 & 0.44895 & 0.59225 & 0.69703 \\
\hline $\mathrm{A} 4$ & D1 & 0.39601 & 0.39896 & 0.40961 \\
\hline A4 & D2 & 0.71469 & 0.75043 & 0.75421 \\
\hline A4 & D3 & 0.54405 & 0.5557 & 0.55868 \\
\hline A4 & D4 & 0.9635 & 1.04212 & 1.06753 \\
\hline $\mathrm{A} 4$ & D5 & 0.37904 & 0.36293 & 0.36489 \\
\hline A4 & D6 & 0.97338 & 1.02982 & 1.04195 \\
\hline A4 & D7 & 0.69751 & 0.68503 & 0.67554 \\
\hline A4 & D8 & 0.97673 & 1.01694 & 1.01886 \\
\hline A5 & D1 & 0.24351 & 0.28168 & 0.32123 \\
\hline A5 & D2 & 0.44398 & 0.57321 & 0.63634 \\
\hline A5 & D3 & 0.31796 & 0.38903 & 0.43756 \\
\hline A5 & D4 & 0.68977 & 0.88914 & 0.97201 \\
\hline A5 & D5 & 0.25285 & 0.27156 & 0.29637 \\
\hline A5 & D6 & 0.69085 & 0.87722 & 0.94936 \\
\hline
\end{tabular}

Page 4 of 13 


\begin{tabular}{llccc}
\hline A5 & D7 & 0.5206 & 0.53196 & 0.55418 \\
A5 & D8 & 0.69391 & 0.86927 & 0.9328 \\
A6 & D1 & 0.56279 & 0.5226 & 0.50509 \\
A6 & D2 & 0.8143 & 0.82394 & 0.8159 \\
A6 & D3 & 0.65507 & 0.63271 & 0.61844 \\
A6 & D4 & 1.07097 & 1.13273 & 0.61844 \\
A6 & D5 & 0.5486 & 0.4968 & 0.47243 \\
A6 & D6 & 1.07464 & 1.12232 & 1.12199 \\
A6 & D7 & 0.62582 & 0.60837 & 0.61085 \\
A6 & D8 & 1.08323 & 1.11778 & 1.10808 \\
A7 & D1 & 0.14794 & 0.18975 & 0.23774 \\
A7 & D2 & 0.2856 & 0.43596 & 0.5292 \\
A7 & D3 & 0.18805 & 0.26555 & 0.32947 \\
A7 & D4 & 0.4985 & 0.74648 & 0.87325 \\
A7 & D5 & 0.16268 & 0.1939 & 0.22982 \\
A7 & D6 & 0.50135 & 0.73846 & 0.85543 \\
A7 & D7 & 0.34927 & 0.38679 & 0.43102 \\
A7 & D8 & 0.50875 & 0.73872 & 0.84724 \\
A8 & D1 & 0.65564 & 0.60487 & 0.5752 \\
A8 & D2 & 0.75121 & 0.743 & 0.73923 \\
A8 & D3 & 0.68308 & 0.64309 & 0.62045 \\
A8 & D4 & 0.94569 & 1.00339 & 1.03284 \\
A8 & D5 & 0.67276 & 0.58135 & 0.55519 \\
A8 & D6 & 0.90554 & 0.95014 & 0.97376 \\
A8 & D7 & 0.86772 & 0.81204 & 0.77516 \\
A8 & D8 & 0.87762 & 0.91648 & 0.93785 \\
\hline
\end{tabular}

Page 5 of 13 
Table S4. Solubility experimental data at 298.2, 308.2, and 318.2 K.

\begin{tabular}{|c|c|c|c|c|}
\hline No. & $m / g$ & $V_{\mathrm{L}} / \mathrm{mL}$ & $c / \mathrm{mol} \cdot \mathrm{L}^{-1}$ & $P / \mathrm{kPa}$ \\
\hline \multicolumn{5}{|c|}{$T=298.2 \mathrm{~K}$} \\
\hline 1 & 3.934 & 4.325 & 0.6549 & 1.5 \\
\hline 2 & 3.980 & 4.379 & 0.7237 & 1.7 \\
\hline 3 & 4.211 & 4.629 & 0.9457 & 2.2 \\
\hline 4 & 4.040 & 4.442 & 1.1167 & 2.5 \\
\hline 5 & 4.121 & 4.530 & 1.3431 & 3.1 \\
\hline \multicolumn{5}{|c|}{$T=308.2 \mathrm{~K}$} \\
\hline 6 & 4.201 & 4.647 & 0.5439 & 1.8 \\
\hline 7 & 4.143 & 4.580 & 0.6926 & 2.2 \\
\hline 8 & 3.921 & 4.337 & 0.9213 & 3.0 \\
\hline 9 & 3.980 & 4.403 & 0.9724 & 3.2 \\
\hline 10 & 4.081 & 4.514 & 1.0656 & 3.4 \\
\hline \multicolumn{5}{|c|}{$T=318.2 \mathrm{~K}$} \\
\hline 11 & 4.209 & 4.685 & 0.5750 & 2.2 \\
\hline 12 & 4.623 & 5.153 & 0.6371 & 2.4 \\
\hline 13 & 3.721 & 4.149 & 0.7659 & 2.8 \\
\hline 14 & 4.404 & 4.908 & 0.9169 & 3.4 \\
\hline 15 & 4.232 & 4.718 & 1.0034 & 3.8 \\
\hline
\end{tabular}




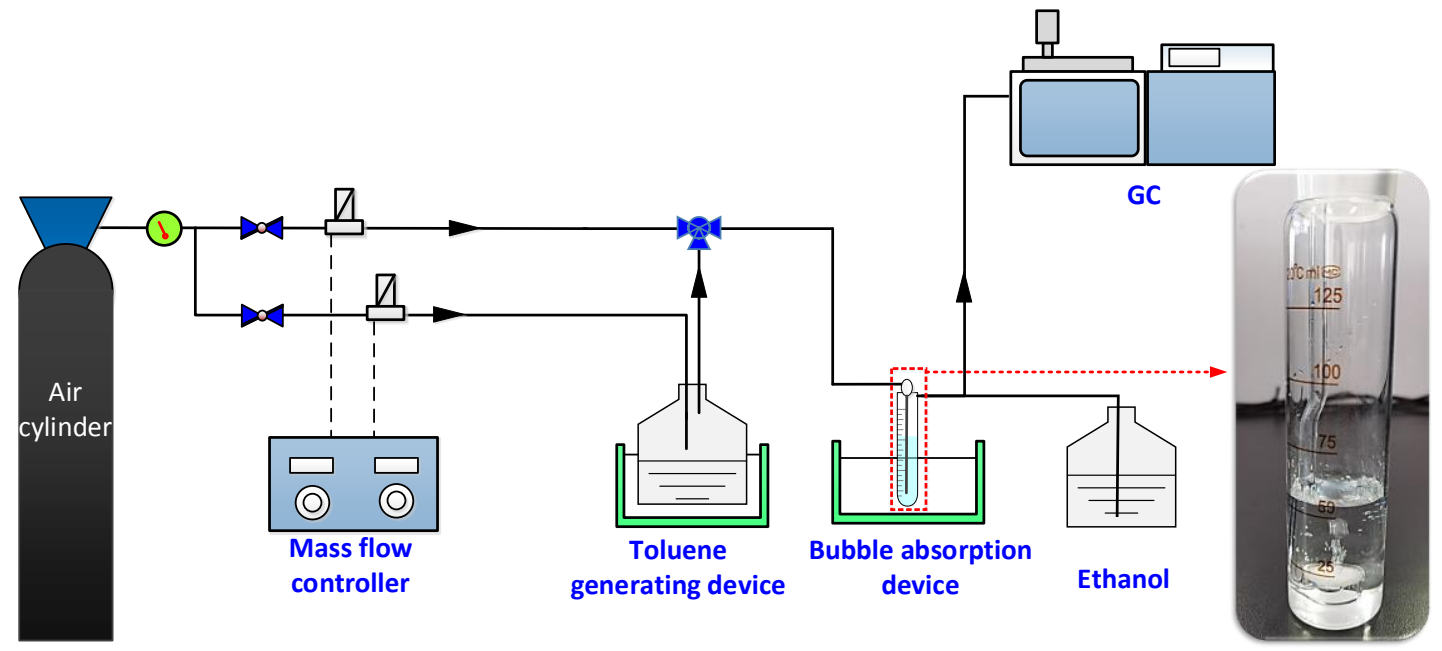

Figure S1. Schematic diagram of the dynamic absorption experiment device 


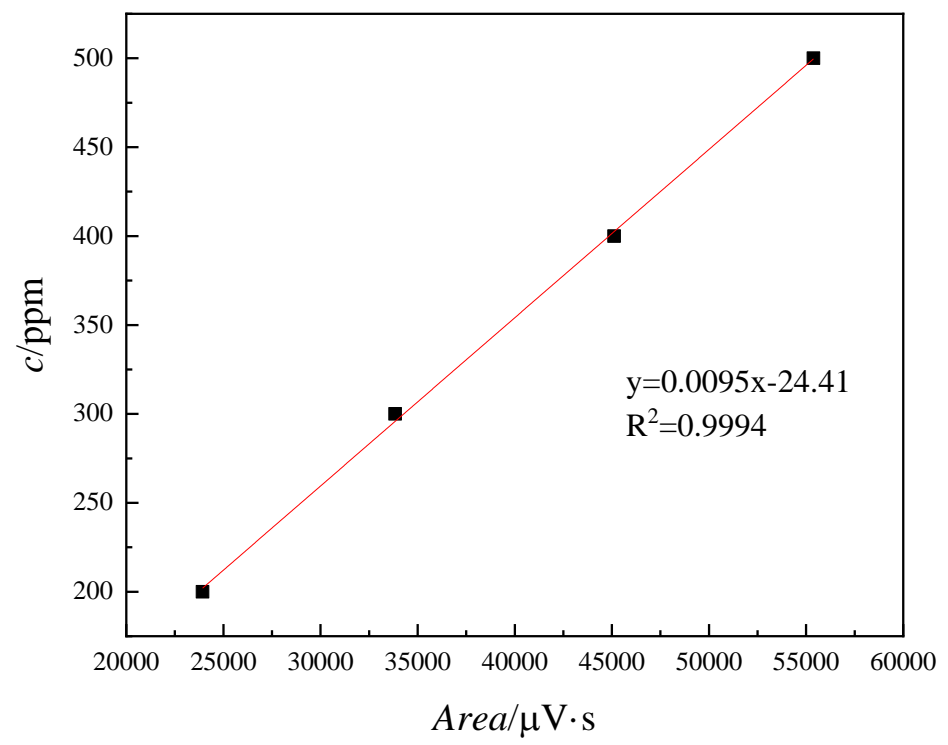

Figure S2. Calibration curve for toluene concentration 

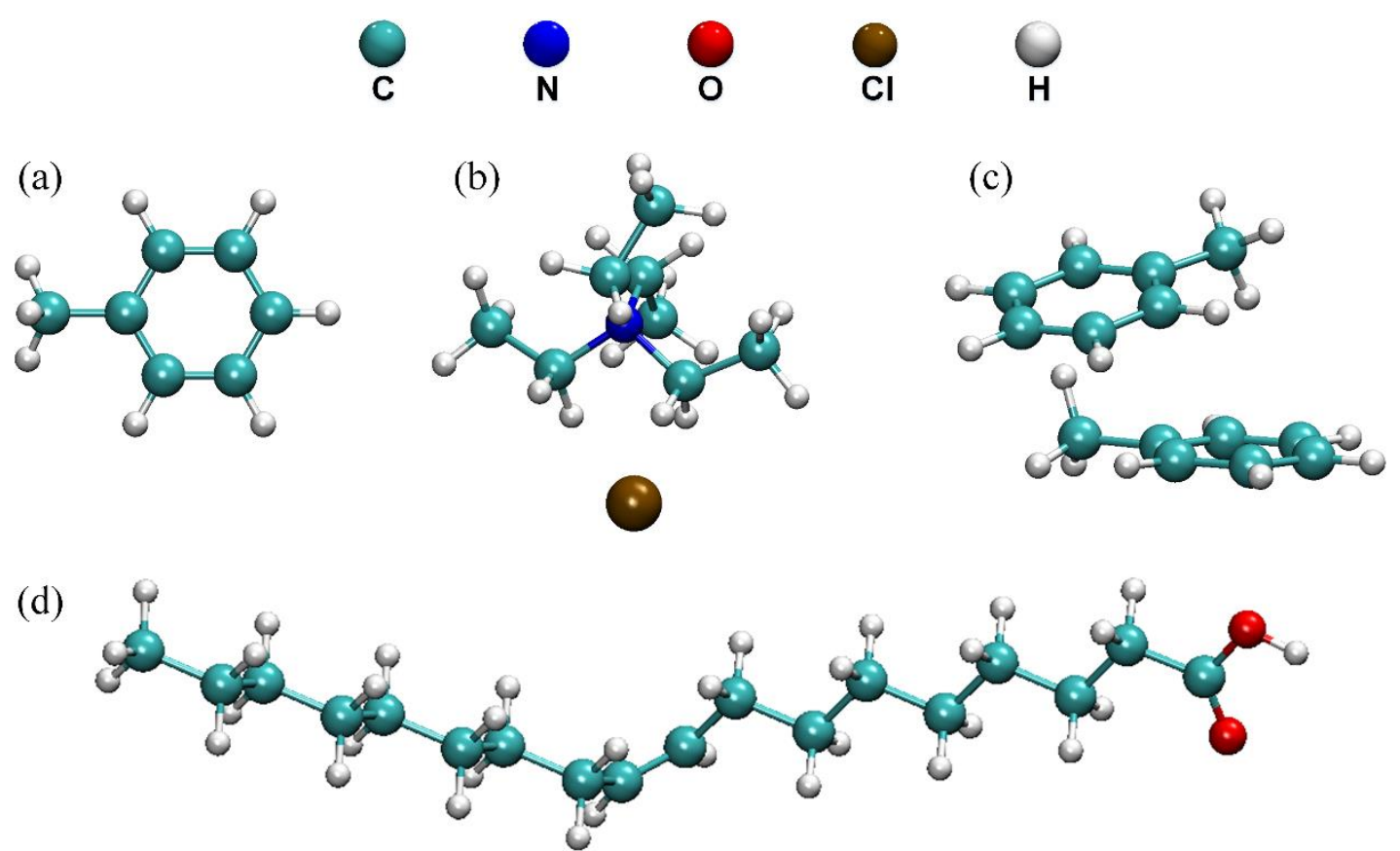

Figure S3. Optimized structures for (a) toluene, (b) TEACl, (c) toluene + toluene, and (d) OA. 


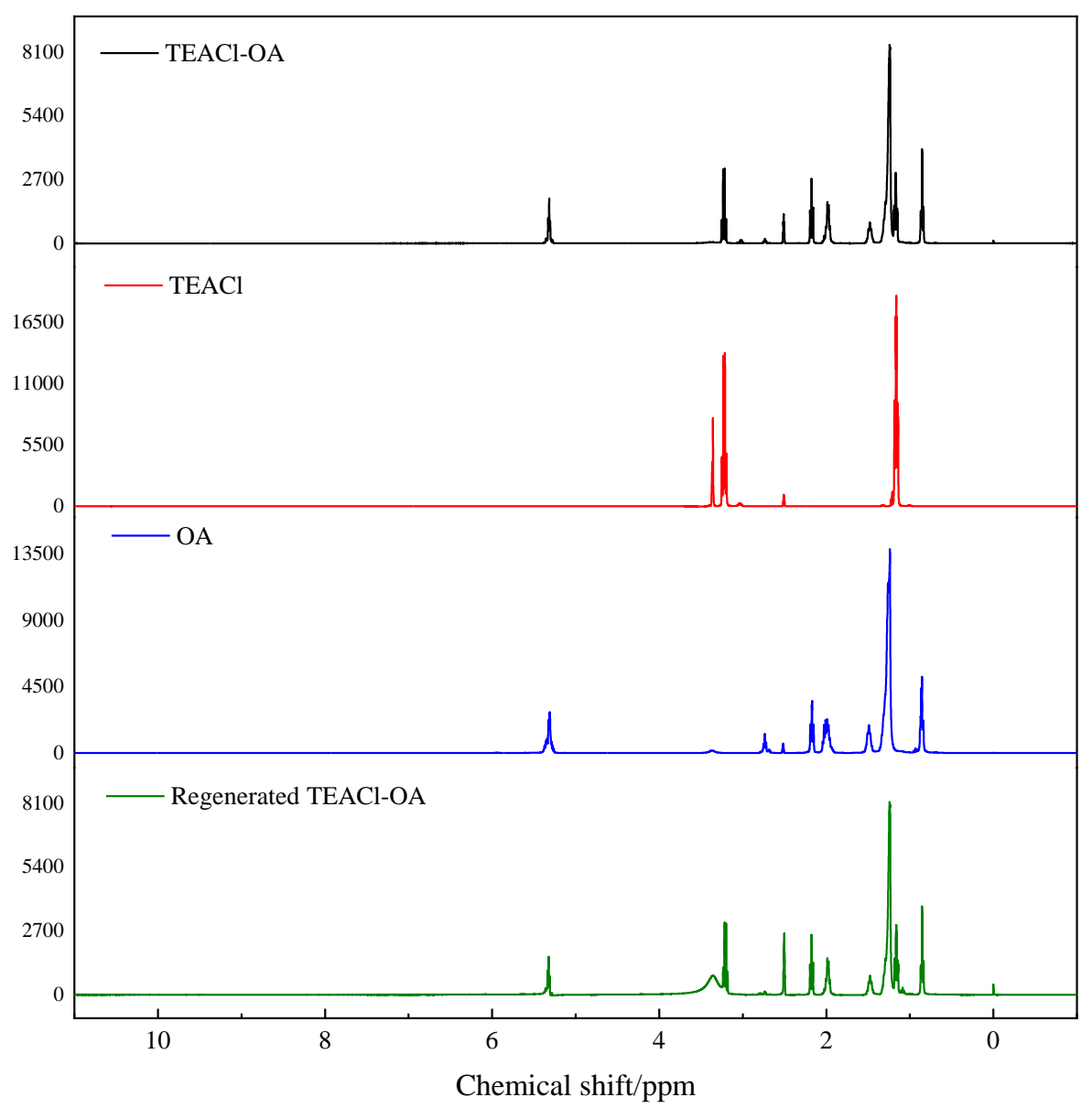

Figure S4. ${ }^{1} \mathrm{H}$ NMR spectra of TEACl-OA, TEACl, OA and regenerated TEACl-OA. 


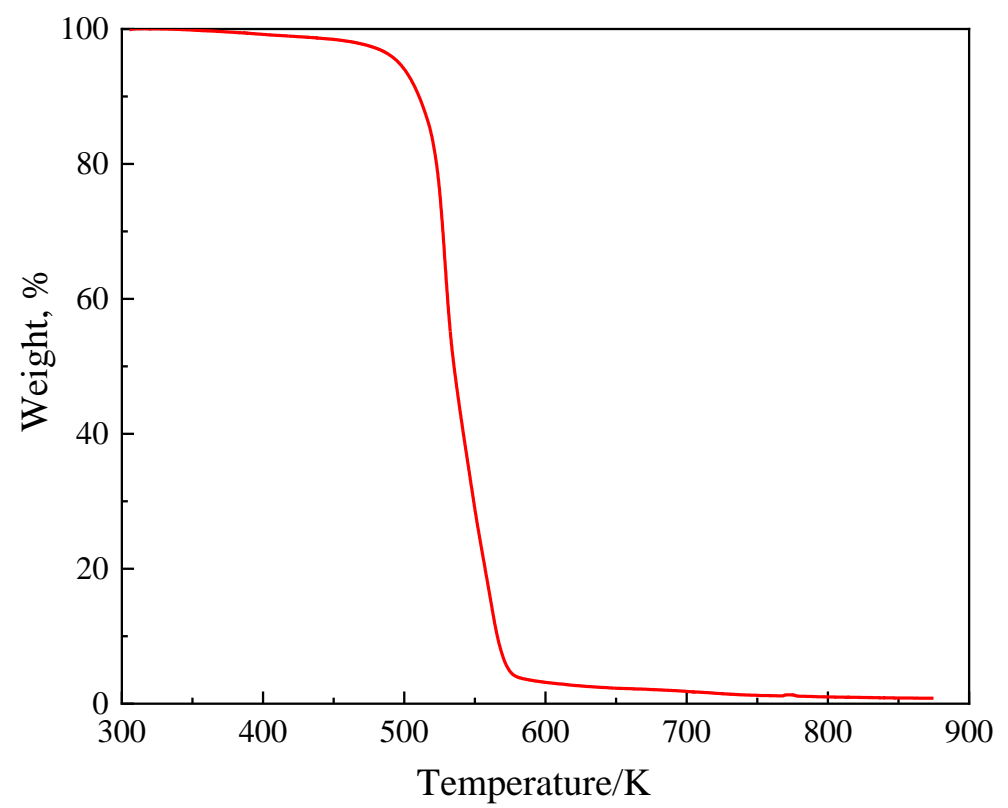

Figure S5. Thermal gravity analysis of TEACl-OA

Page 11 of 13 


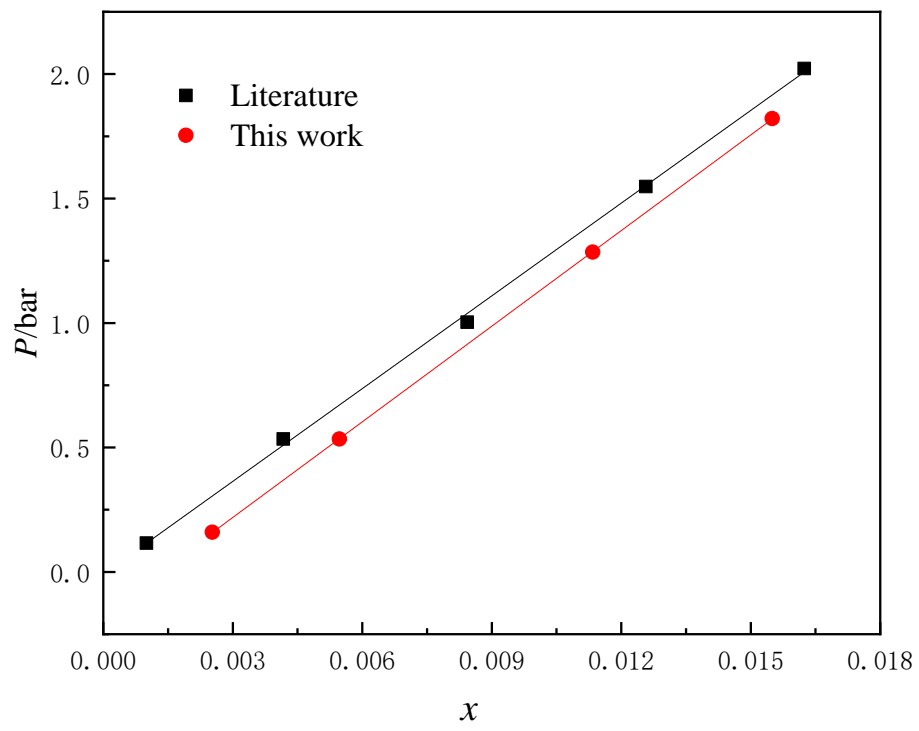

Figure S6. Feasibility verification of the solubility experimental device by comparing experimental data in this wok and the literature result ${ }^{1}$. 


\section{REFERENCES}

R1. Liu, F.; Chen, W.; Mi, J.; Zhang, J; Kan, X.; Zhong, F; Huang, K.; Zheng, A;

Jiang, L. Thermodynamic and molecular insights into the absorption of $\mathrm{H}_{2} \mathrm{~S}, \mathrm{CO}_{2}$, and $\mathrm{CH}_{4}$ in choline chloride plus urea mixtures. AIChE J., 2019, 65, 16574. 